Bull. Korean Math. Soc. 47 (2010), No. 3, pp. 563-573

DOI 10.4134/BKMS.2010.47.3.563

\title{
NEW LOOK AT THE CONSTRUCTIONS OF MULTIWAVELET FRAMES
}

\author{
Hong Oh Kim, Rae Young Kim, and Jae Kun Lim
}

\begin{abstract}
Using the fiberization technique of a shift-invariant space and the matrix characterization of the decomposition of a shift-invariant space of finite length into an orthogonal sum of singly generated shift-invariant spaces, we show that the main result in [13] can be interpreted as a statement about the length of a shift-invariant space, and give a more natural construction of multiwavelet frames from a frame multiresolution analysis of $L^{2}\left(\mathbb{R}^{d}\right)$.
\end{abstract}

\section{Introduction}

For $y \in \mathbb{R}^{d}$ and $f \in L^{2}\left(\mathbb{R}^{d}\right), T_{y}$ denotes the unitary translation operator such that $T_{y} f(x):=f(x-y)$ and $D$ denotes the unitary dyadic dilation operator such that $D f(x):=2^{d / 2} f(2 x)$. A family of closed subspaces $\left\{V_{k}\right\}_{k \in \mathbb{Z}}$ of $L^{2}\left(\mathbb{R}^{d}\right)$ is said to be a frame multiresolution analysis (FMRA) if

(1) $V_{k} \subset V_{k+1}, \quad k \in \mathbb{Z}$;

(2) $\overline{\cup_{k \in \mathbb{Z}} V_{k}}=L^{2}\left(\mathbb{R}^{d}\right), \cap_{k \in \mathbb{Z}} V_{k}=\{0\}$;

(3) $D\left(V_{k}\right)=V_{k+1}, \quad k \in \mathbb{Z}$;

(4) There exists a scaling function $\varphi \in V_{0}$ such that $\left\{T_{k} \varphi: k \in \mathbb{Z}^{d}\right\}$ is a tight frame with the frame bound one for $V_{0}$.

For the sake of brevity of presentation we only consider the dyadic dilation even though all of our results can be repeated verbatim if we consider general dilation, and we do not recount the theory and the history of FMRA in the literature $[1,2,7,9,10,11,12,13]$.

It is well-known that the condition (4) is equivalent to the condition that $\left\{T_{k} \varphi: k \in \mathbb{Z}^{d}\right\}$ is a frame for $V_{0}[3,14]$. Moreover, let $W_{0}:=V_{1} \ominus V_{0}$ and $W_{j}:=D^{j}\left(W_{0}\right)$. Then it is easy to see that $L^{2}\left(\mathbb{R}^{d}\right)=\oplus_{j \in \mathbb{Z}} W_{j}$. Hence, if there exists $\left\{\psi_{1}, \psi_{2}, \ldots, \psi_{L}\right\} \subset W_{0}$ such that $\left\{T_{k} \psi_{l}: k \in \mathbb{Z}^{d}, 1 \leq l \leq L\right\}$ is a frame for $W_{0}$, then $\left\{D^{j} T_{k} \psi_{l}: j \in \mathbb{Z}, k \in \mathbb{Z}^{d}, 1 \leq l \leq L\right\}$ is a frame for $L^{2}\left(\mathbb{R}^{d}\right)$. In this case we say that $\left\{\psi_{1}, \psi_{2}, \ldots, \psi_{L}\right\}$ is a (semi-orthogonal) wavelet set. It

Received December 13, 2008; Revised March 10, 2009.

2000 Mathematics Subject Classification. 42C15, 42C40.

Key words and phrases. wavelet, frame, multiresolution analysis, shift-invariant space.

This research was supported by the Yeungnam University research grants in 2008 . 
is interesting to determine the minimal cardinality of a wavelet set, which is called the index of an FMRA in [13]. It is well-known and easy to see that the index is $2^{d}-1$ if the shifts (multi-integer translates) of a scaling function form a Riesz basis of $V_{0}$.

We now present the main result in [13]. For $f \in L^{2}\left(\mathbb{R}^{d}\right)$ and $x \in \mathbb{R}^{d}$, let $\hat{f}_{\| x}:=(\hat{f}(x+k))_{k \in \mathbb{Z}^{d}}$. Here $\wedge$ denotes the Fourier transform defined by $\hat{f}(x):=\int_{\mathbb{R}^{d}} f(t) e^{-2 \pi i x \cdot t} d t$ for $f \in L^{1}\left(\mathbb{R}^{d}\right) \cap L^{2}\left(\mathbb{R}^{d}\right)$, and extended to be a unitary operator on $L^{2}\left(\mathbb{R}^{d}\right)$ by the Plancherel theorem. Then $\hat{f}_{\| x} \in \ell^{2}\left(\mathbb{Z}^{d}\right)$ for a.e. $x \in \mathbb{R}^{d}$. Suppose we are given an FMRA with a scaling function $\varphi$. Let $\Phi(x):=\left\|\hat{\varphi}_{\| x}\right\|_{\ell^{2}\left(\mathbb{Z}^{d}\right)}^{2}, \mathcal{Q}:=\left\{x \in \mathbb{R}^{d}: \Phi(2 x)=0\right\}$, and

$$
Q:=\left\{q_{1}, q_{2}, \ldots, q_{2^{d}}\right\}:=\{0,1\}^{d},
$$

which is a complete set of representatives of the quotient group $\mathbb{Z}^{d} / 2 \mathbb{Z}^{d}$.

For a Lebesgue measurable subset $A$ of $\mathbb{R}^{d}|A|$ denotes its Lebesgue measure.

Definition 1.1. (i) For a fixed point $x \in \mathbb{R}^{d}$, if the sequence $\{\Phi(x+q / 2): q \in$ $Q\}$ has just $r$ nonzero terms, then we say $x \in B_{r}$.

(ii) If an integer $p$ satisfies both $\left|B_{p}\right|>0$ and $\left|B_{r}\right|=0(r>p)$, then we define the index $\lambda$ of the underlying FMRA as follows:

$$
\lambda= \begin{cases}p, & \text { if }\left|\mathcal{Q} \cap B_{p}\right|>0 \\ p-1, & \text { if }\left|\mathcal{Q} \cap B_{p}\right|=0\end{cases}
$$

The following is the main result in [13].

Theorem $1.2([13])$. Let $\left\{V_{j}\right\}_{j \in \mathbb{Z}}$ be an FMRA for $L^{2}\left(\mathbb{R}^{d}\right)$ with its index $\lambda$. Then there exists a wavelet set $\left\{\psi_{l}\right\}_{l=1}^{n} \subset W_{0}$ such that $\left\{T_{k} \psi_{l}: 1 \leq l \leq n, k \in\right.$ $\left.\mathbb{Z}^{d}\right\}$ is a tight frame for $W_{0}$ with the frame bound one if and only if $n \geq \lambda$. In this case, $\left\{D^{j} T_{k} \psi_{l}: j \in \mathbb{Z}, k \in \mathbb{Z}^{d}, 1 \leq l \leq n\right\}$ is a tight frame for $L^{2}\left(\mathbb{R}^{d}\right)$ with the frame bound one.

Theorem 1.2 has a most natural interpretation in the language of the theory of shift-invariant spaces $[3,4,5,6,14,15]$. A closed subspace $S$ of $L^{2}\left(\mathbb{R}^{d}\right)$ is said to be a shift-invariant space if it is invariant under each shift. In this case, $\hat{S}_{\| x}:=\left\{\hat{f}_{\| x}: f \in S\right\}$, called the fiber of $S$ at $x$, is a closed subspace of $\ell^{2}\left(\mathbb{Z}^{d}\right)$ for a.e. $x \in \mathbb{T}^{d}:=\mathbb{R}^{d} / \mathbb{Z}^{d} \equiv[0,1]^{d} \equiv[-1 / 2,1 / 2]^{d}$. The spectrum of $S$ is defined to be $\sigma(S):=\left\{x \in \mathbb{T}^{d}: \hat{S}_{\| x} \neq\{0\}\right\}$. It is known that any shift-invariant space $S$ has a generating set $F \subset L^{2}\left(\mathbb{R}^{d}\right)$, which is at most countable, such that $S=\mathcal{S}(F):=\overline{\operatorname{span}}\left\{T_{k} f: k \in \mathbb{Z}^{d}, f \in F\right\}$. The length of $S$ is defined to be len $S:=\min \{\# F: S=\mathcal{S}(F)\}$. Moreover, we have $([3])$

$$
\text { len } S=\operatorname{ess-sup}\left\{\operatorname{dim} \hat{S}_{\| x}: x \in \mathbb{T}^{d}\right\} .
$$

Now, suppose $L:=\operatorname{len} S$ is finite. Then, a careful examination of [4, Theorem 3.3] shows the following fact: For any $F \subset L^{2}\left(\mathbb{R}^{d}\right)$ with $\# F<L$, $S \neq \mathcal{S}(F)$, and for any $n \geq L$ there exists $F \subset L^{2}\left(\mathbb{R}^{d}\right)$ with $\# F=n$ such 
that $S=\oplus_{f \in F} \mathcal{S}(\{f\})$, and $\left\{T_{k} f: k \in \mathbb{Z}^{d}, f \in F\right\}$ is a tight frame for $S$ with the bound one. Here $\oplus$ denotes the orthogonal sum. Note that $V_{0}$ is a shift-invariant space with len $V_{0}=1$. It is a standard fact that $V_{1}$ is also a shift-invariant space with len $V_{1} \leq 2^{d}[7,11]$ (see Section 2), and hence $W_{0}$ is also a shift-invariant space with len $W_{0} \leq 2^{d}$. Therefore, once we show that the index $\lambda$ and len $W_{0}$ coincide (Theorem 2.4), then Theorem 1.2 follows. We also give a more natural construction of a wavelet set in Theorem 2.5 using the fiberization technique of shift-invariant space theory, the newly found matrix characterization theorem of the decomposition of a shift-invariant space of finite length into an orthogonal sum of singly generated shift-invariant spaces (Theorem 2.2) and elementary linear algebra.

\section{Main results}

In this section, we prove that the index of an FMRA is len $W_{0}$, and construct a semi-orthogonal wavelet set from an FMRA using the language of shift-invariant spaces.

Suppose that $\varphi$ is a scaling function of an FMRA $\left\{V_{j}\right\}_{j \in \mathbb{Z}}$. We assume that $\sum_{k \in \mathbb{Z}^{d}}|\hat{\varphi}(x+k)|^{2}=\chi_{\sigma\left(V_{0}\right)}(x)$ a.e. $x \in \mathbb{T}^{d}$, which is equivalent to the condition that the shifts of $\varphi$ form a tight frame with the frame bound one for $V_{0}[3,14]$. Since $V_{0} \subset V_{1}:=D\left(V_{0}\right), \varphi$ is refinable, i.e., there exists $m_{0} \in L^{2}\left(\mathbb{T}^{d}\right)$ such that

$$
\hat{\varphi}(2 x)=m_{0}(x) \hat{\varphi}(x) \quad \text { a.e. } x \in \mathbb{R}^{d} .
$$

We note that each $k \in \mathbb{Z}^{d}$ can be written uniquely as $k=2 k^{\prime}+q$ for some $k^{\prime} \in \mathbb{Z}^{d}$ and $q \in Q$. Note also that $D T_{y}=T_{y / 2} D$ for each $y \in \mathbb{R}^{d}$. Therefore, $\left\{D T_{k} \varphi: k \in \mathbb{Z}^{d}\right\}=\left\{T_{k^{\prime}} D T_{q} \varphi: k^{\prime} \in \mathbb{Z}^{d}, q \in Q\right\}$. Hence $V_{1}=\mathcal{S}(\Pi)$, where $\Pi:=\left\{D T_{q} \varphi: q \in Q\right\}$. This shows that len $V_{1} \leq 2^{d}$.

We recall the known facts about the fibers of the shift-invariant subspaces $V_{0}, W_{0}$ and $V_{1}$ in $[7,8,11]$ that will be used throughout this article. For $q \in Q$, define $P_{q}: \ell^{2}\left(\mathbb{Z}^{d}\right) \rightarrow \ell^{2}\left(\mathbb{Z}^{d}\right)$ via

$$
\left(P_{q} a\right)(k):= \begin{cases}a(k), & \text { if } k \in 2 \mathbb{Z}^{d}+q, \\ 0, & \text { otherwise. }\end{cases}
$$

Then $\ell^{2}\left(\mathbb{Z}^{d}\right)=\oplus_{q \in Q} P_{q}\left(\ell^{2}\left(\mathbb{Z}^{d}\right)\right)$. Define, for each $x \in \mathbb{T}^{d}$ and each $q \in Q$,

$$
a_{x, q}:=P_{q}\left(\left(\hat{\varphi}\left(\frac{x+k}{2}\right)\right)_{k \in \mathbb{Z}^{d}}\right) .
$$

Note that $a_{x, q}$ is the 'up-sampled' version of $\hat{\varphi}_{\|(x+q) / 2}$, i.e.,

$$
\left\{\begin{array}{llll}
a_{x, q}(2 k+q) & =\hat{\varphi}_{\|(x+q) / 2}(k), & & \text { if } k \in \mathbb{Z}^{d}, \\
a_{x, q}(k) & =0, & & \text { if } k \notin 2 \mathbb{Z}^{d}+q .
\end{array}\right.
$$

Therefore,

$$
\left\|a_{x, q}\right\|_{\ell^{2}\left(\mathbb{Z}^{d}\right)}=\left\|\hat{\varphi}_{\|(x+q) / 2}\right\|_{\ell^{2}\left(\mathbb{Z}^{d}\right)} .
$$


With this notation, we get

$$
\begin{aligned}
\hat{\varphi}_{\| x} & =(\hat{\varphi}(x+k))_{k \in \mathbb{Z}^{d}}=\left(m_{0}\left(\frac{x+k}{2}\right) \hat{\varphi}\left(\frac{x+k}{2}\right)\right)_{k \in \mathbb{Z}^{d}} \\
& =\sum_{q \in Q} m_{0}\left(\frac{x+q}{2}\right) a_{x, q} .
\end{aligned}
$$

Then, we have, by (6) and the orthogonality of $\left\{a_{x, q}: q \in Q\right\}$,

$$
\chi_{\sigma\left(V_{0}\right)}(x)=\sum_{k \in \mathbb{Z}^{d}}|\hat{\varphi}(x+k)|^{2}=\sum_{q \in Q}\left|m_{0}\left(\frac{x+q}{2}\right)\right|^{2}\left\|a_{x, q}\right\|_{\ell^{2}\left(\mathbb{Z}^{d}\right)}^{2} .
$$

The basic facts about the fibers of the shift-invariant spaces $V_{0}, W_{0}$ and $V_{1}$ are concisely represented in the following lemma whose proof can be found in $[7,8,11]$.

Lemma 2.1 ([7, 8, 11]). For a.e. $x \in \mathbb{T}^{d}$

$$
\begin{aligned}
& \hat{V}_{0 \| x}=\operatorname{span}\left\{\sum_{q \in Q} m_{0}\left(\frac{x+q}{2}\right) a_{x, q}\right\}, \\
& \hat{V}_{1 \| x}=\operatorname{span}\left\{a_{x, q}: q \in Q\right\} .
\end{aligned}
$$

In particular, for a.e. $x \in \mathbb{T}^{d}$,

$$
\operatorname{dim} \hat{V}_{1|| x}=\#\left\{a_{x, q}: a_{x, q} \neq 0\right\} .
$$

Now, suppose that $\left\{\psi_{1}, \psi_{2}, \ldots, \psi_{n}\right\} \subset V_{1}$. Let $W_{0}^{(i)}:=\mathcal{S}\left(\left\{\psi_{i}\right\}\right)$ for $i=$ $1,2, \ldots, n$. For the sake of notation let $\psi_{0}:=\varphi$ and $W_{0}^{(0)}:=V_{0}$. Then there exist $m_{1}, m_{2}, \ldots, m_{n} \in L^{2}\left(\mathbb{T}^{d}\right)$ such that

$$
\hat{\psi}_{i}(2 x):=m_{i}(x) \hat{\varphi}(x), 1 \leq i \leq n .
$$

Then for each $i=1,2, \ldots, n$,

$$
\hat{\psi}_{i \| x}=\left(\hat{\psi}_{i}(x+k)\right)_{k \in \mathbb{Z}^{d}}=\sum_{q \in Q} m_{i}\left(\frac{x+q}{2}\right) a_{x, q} .
$$

Let $M$ be the matrix-valued function defined by

$$
M(x):=\left(\begin{array}{cccc}
m_{0}\left(x+\frac{q_{1}}{2}\right) & m_{0}\left(x+\frac{q_{2}}{2}\right) & \cdots & m_{0}\left(x+\frac{q_{2} d}{2}\right) \\
m_{1}\left(x+\frac{q_{1}}{2}\right) & m_{1}\left(x+\frac{q_{2}}{2}\right) & \cdots & m_{1}\left(x+\frac{q_{2} d}{2}\right) \\
\vdots & \vdots & \ddots & \vdots \\
m_{n}\left(x+\frac{q_{1}}{2}\right) & m_{n}\left(x+\frac{q_{2}}{2}\right) & \cdots & m_{n}\left(x+\frac{q_{2} d}{2}\right)
\end{array}\right)
$$


and let $M(x)^{k_{1}, \ldots, k_{l}}$ denote the matrix-valued function obtained from $M(x)$ by deleting the $k_{1}, \ldots, k_{l}$-th columns of $M(x)$. Note that $M$ satisfies

$$
\left(\begin{array}{c}
\hat{\varphi}_{|| x} \\
\hat{\psi}_{1 \| x} \\
\vdots \\
\hat{\psi}_{n \| x}
\end{array}\right)=M\left(\frac{x}{2}\right)\left(\begin{array}{c}
a_{x, q_{1}} \\
a_{x, q_{2}} \\
\vdots \\
a_{x, q_{2} d}
\end{array}\right) .
$$

Let us define, for $0 \leq i \leq 2^{d}$,

$$
\Delta_{i}:=\left\{x \in \mathbb{T}^{d}: \operatorname{dim} \hat{V}_{1 \| x}=i\right\},
$$

where $\operatorname{dim} \hat{V}_{1 \| x}$ can be computed by (10). Then $\sigma\left(V_{1}\right)=\uplus_{i=1}^{2^{d}} \Delta_{i}$, where $\uplus$ denotes the disjoint union. And, for each $1 \leq l \leq 2^{d}-1$ and each $k_{1}, k_{2}, \ldots, k_{l}$ such that $1 \leq k_{1}<k_{2}<\cdots<k_{l} \leq 2^{d}$, define

$$
\Delta_{2^{d}-l}^{k_{1}, \ldots, k_{l}}:=\left\{x \in \Delta_{2^{d}-l}: a_{x, q_{k_{1}}}=\cdots=a_{x, q_{k_{l}}}=0\right\},
$$

which is clearly a Lebesgue measurable set. Then we have, by (10) and the orthogonality of $\left\{a_{x, q}: q \in Q\right\}$,

$$
\Delta_{2^{d}-l}=\biguplus_{1 \leq k_{1}<k_{2}<\cdots<k_{l} \leq 2^{d}} \Delta_{2^{d}-l}^{k_{1}, k_{2}, \ldots, k_{l}}
$$

The following matrix characterization theorem of the decomposition of a shift-invariant space of finite length into an orthogonal sum of singly generated shift-invariant spaces is used repeatedly in the construction of a wavelet set.

Theorem 2.2. Let $\psi_{0}:=\varphi \in L^{2}\left(\mathbb{R}^{d}\right)$ be refinable and its shifts form a tight frame with the frame bound one for $V_{0}:=W_{0}^{(0)}:=\mathcal{S}(\{\varphi\})$. Let $m_{1}, \ldots, m_{n}$ be in $L^{2}\left(\mathbb{T}^{d}\right)$ and define $\psi_{1}, \ldots, \psi_{n}$ and $M$ as in (11) and (13), respectively. Then the shifts of $\psi_{i}$ form a tight frame with the frame bound one for $W_{0}^{(i)}:=\mathcal{S}\left(\left\{\psi_{i}\right\}\right)$ for each $i=1,2, \ldots, n$ and

$$
V_{1}:=D V_{0}=W_{0}^{(0)} \oplus W_{0}^{(1)} \oplus \cdots \oplus W_{0}^{(n)}
$$

if and only if

(1) For a.e. $x \in \Delta_{2^{d}}, 2^{d}$ rows of $M(x / 2)$ are orthonormal vectors and the remaining rows are zero vectors in $\mathbb{C}^{2^{d}}$;

(2) For each $l=1,2, \ldots, 2^{d}-1$ and for each choice of $1 \leq k_{1}<k_{2}<\cdots<$ $k_{l} \leq 2^{d}, 2^{d}-l$ rows of $M(x / 2)^{k_{1}, \ldots, k_{l}}$ are orthonormal vectors and the remaining rows are zero vectors in $\mathbb{C}^{2^{d}-l}$ for a.e. $x \in \Delta_{2^{d}-l}^{k_{1}, \ldots, k_{l}}$.

We can check $\psi_{j} \in L^{2}\left(\mathbb{R}^{d}\right), 1 \leq j \leq n$, by (7) and (11). For the proof of Theorem 2.2, we need the following lemma, whose 'only if' part is mentioned in [3].

Lemma 2.3. Let $A, B$ and $C$ be shift-invariant subspaces of $L^{2}\left(\mathbb{R}^{d}\right)$. Then $A=B \oplus C$ if and only if $\hat{A}_{\| x}=\hat{B}_{\| x} \oplus \hat{C}_{\| x}$ for a.e. $x \in \mathbb{T}^{d}$. 
Proof. $(\Rightarrow)$ : Let $b \in B$ and $c \in C$. Then, for any $k \in \mathbb{Z}^{d}$,

$$
0=\left\langle b, T_{k} c\right\rangle_{L^{2}\left(\mathbb{R}^{d}\right)}=\int_{\mathbb{T}^{d}}\left\langle\hat{b}_{\| x}, \hat{c}_{\| x}\right\rangle_{\ell^{2}\left(\mathbb{Z}^{d}\right)} e^{2 \pi i k \cdot x} d x .
$$

This shows that, for any $b \in B$ and $c \in C,\left\langle\hat{b}_{\| x}, \hat{c}_{\| x}\right\rangle_{\ell^{2}\left(\mathbb{Z}^{d}\right)}=0$ for a.e. $x \in \mathbb{T}^{d}$. Now, let $a \in A$. Then there exist $b \in B$ and $c \in C$ such that $a=b \oplus c$. Hence, for a.e. $x \in \mathbb{T}^{d}, \hat{a}_{\| x}=\hat{b}_{\| x} \oplus \hat{c}_{|| x}$.

$(\Leftarrow)$ : The assumption implies that $B \perp C$ by a calculation similar to the previous one. Since $B$ and $C$ are shift-invariant subspaces, so is $D:=B \oplus C$. Now, the fiber spaces of $A$ and $D$ coincide a.e.. Hence $A=D$ by a standard result (see, for example, [4, Proposition 1.5]).

Proof of Theorem 2.2. $(\Rightarrow)$ : The tight frame assumption implies that

$$
\sum_{k \in \mathbb{Z}}\left|\hat{\psi}_{i}(x+k)\right|^{2}=\chi_{\sigma\left(W_{0}^{(i)}\right)}(x) \text { a.e. for each } i=0,1, \ldots, n \text {. }
$$

In particular, the tight frame assumption for $\psi_{0}=\varphi$ implies that $\left\|a_{x, q}\right\|_{\ell^{2}\left(\mathbb{Z}^{d}\right)}^{2}=$ 1 or 0 a.e. by (5) for each $q \in Q$. We have

$$
\chi_{\sigma\left(W_{0}^{(i)}\right)}(x)=\sum_{q \in Q}\left|m_{i}\left(\frac{x+q}{2}\right)\right|^{2}\left\|a_{x, q}\right\|_{\ell^{2}\left(\mathbb{Z}^{d}\right)}^{2}=\sum_{q \in Q, a_{x, q} \neq 0}\left|m_{i}\left(\frac{x+q}{2}\right)\right|^{2}
$$

for a.e. $x \in \mathbb{T}^{d}$ and for each $i=0,1, \ldots, n$ by Equations (6) and (12) and by the orthogonality of $\left\{a_{x, q}: q \in Q\right\}$. On the other hand, Equations (16), (6) and (12) imply that

(18) $0=\sum_{q \in Q} m_{i}\left(\frac{x+q}{2}\right) \overline{m_{j}}\left(\frac{x+q}{2}\right)\left\|a_{x, q}\right\|_{\ell^{2}\left(\mathbb{Z}^{d}\right)}^{2}=\sum_{q \in Q, a_{x, q} \neq 0} m_{i}\left(\frac{x+q}{2}\right) \overline{m_{j}}\left(\frac{x+q}{2}\right)$

for a.e. $x \in \mathbb{T}^{d}$ and for each $0 \leq i \neq j \leq n$. Now, by Lemma 2.3, (16) is equivalent to the fact that, for a.e. $x \in \mathbb{T}^{d}$,

$$
\hat{V}_{1|| x}=\left(W_{0}^{(0)}\right)_{\| x}^{\wedge} \oplus\left(W_{0}^{(1)}\right)_{\| x}^{\wedge} \oplus \cdots \oplus\left(W_{0}^{(n)}\right)_{\| x}^{\wedge} .
$$

Therefore, by Lemma 2.1 and (19), we have

$$
\operatorname{dim} \hat{V}_{1|| x}=\#\left\{q \in Q: a_{x, q} \neq 0\right\}=\#\left\{i \in\{0,1, \ldots, n\}: x \in \sigma\left(W_{0}^{(i)}\right)\right\} .
$$

Suppose that $x \in \Delta_{2^{d}-l}^{k_{1}, \ldots, k_{l}}$. For a.e. such $x, \operatorname{dim} \hat{V}_{1 \| x}=2^{d}-l$ and $a_{x, q}=0$ if $q \in\left\{q_{k_{1}}, \ldots, q_{k_{l}}\right\}$ and $a_{x, q} \neq 0$ if $q \in Q \backslash\left\{q_{k_{1}}, \ldots, q_{k_{l}}\right\}$. Hence there exist exactly $2^{d}-l$ number of $i \in\{0,1, \ldots, n\}$ such that $x \in \sigma\left(W_{0}^{(i)}\right)$. Now, Equations (17) and (18) imply that those $2^{d}-l$ rows of $M(x / 2)^{k_{1}, \ldots, k_{l}}$ is orthonormal vectors in $\mathbb{C}^{2^{d}-l}$ and the remaining rows are zero vectors. This proves Condition (2). Condition (1) can be proved similarly.

$(\Leftarrow)$ : Note that Condition (11) implies that $\left\{\psi_{i}\right\}_{i=1}^{n} \subset V_{1}$. We show that (19) holds a.e. $x \in \mathbb{T}^{d}$ and that for $i=1, \ldots, n,\left\|\hat{\psi}_{i \| x}\right\|_{\ell^{2}\left(\mathbb{Z}^{d}\right)}^{2}=0$ or 1 a.e., which is equivalent to the condition that the shifts of $\psi_{i}$ form a tight frame. 
If $x \in \mathbb{T}^{d} \backslash \sigma\left(V_{1}\right)$, then (19) holds trivially. Now let $x \in \Delta_{2^{d}}$. For a.e. such $x,(14)$ holds. The elements of the right-most vector in the right-hand side of (14) form an orthonormal basis of $\hat{V}_{1|| x}$. Condition (1) now implies that $2^{d}$ elements of the vector in the left-hand side of (14) also form an orthonormal basis of $\hat{V}_{1|| x}$ and the remaining elements are zero vectors of $\ell^{2}\left(\mathbb{Z}^{d}\right)$. Hence (19) holds a.e. $x \in \Delta_{2^{d}}$. Moreover, $\left\|\hat{\psi}_{i \| x}\right\|_{\ell^{2}\left(\mathbb{Z}^{d}\right)}^{2}=0$ or 1 for a.e. $x \in \Delta_{2^{d}}$. On the other hand, for a.e. $x \in \Delta_{2^{d}-l}^{k_{1}, \ldots, k_{l}},(14)$ also holds. For those $x \in \Delta_{2^{d}-l}^{k_{1}, \ldots, k_{l}}$, $\left\{a_{x, q}: q \in Q \backslash\left\{q_{k_{1}}, q_{k_{2}}, \ldots, q_{k_{l}}\right\}\right\}$ is an orthonormal basis for $\hat{V}_{1|| x}$, and $a_{x, q}=0$ if $q \in\left\{q_{k_{1}}, q_{k_{2}}, \ldots, q_{k_{l}}\right\}$. Hence, (14) now takes a simpler form:

$$
\left(\left(\hat{\psi}_{i \| x}\right)_{i=0}^{n}\right)^{t}=M(x / 2)^{k_{1}, \ldots, k_{l}}\left(\left(a_{x, q}\right)_{q \in Q \backslash\left\{q_{k_{1}}, q_{k_{2}}, \ldots, q_{k_{l}}\right\}}\right)^{t},
$$

where $t$ denotes the transpose of a matrix or a vector. Now (19) holds by the same argument for $x \in \Delta_{2^{d}}$, and $\left\|\hat{\psi}_{i \| x}\right\|_{\ell^{2}\left(\mathbb{Z}^{d}\right)}^{2}=0$ or 1 for a.e. $x \in \Delta_{2^{d}}^{k_{1}, \ldots, k_{l}}$.

We now show that len $W_{0}$ is equal to the index of the FMRA in Definition 1.1.

Theorem 2.4. Let $\lambda_{1}:=\operatorname{len} V_{1}$. Then

$$
\begin{aligned}
\text { len } W_{0} & = \begin{cases}\lambda_{1}, & \text { if }\left|\Delta_{\lambda_{1}} \backslash \sigma\left(V_{0}\right)\right|>0, \\
\lambda_{1}-1, & \text { if }\left|\Delta_{\lambda_{1}} \backslash \sigma\left(V_{0}\right)\right|=0 .\end{cases} \\
& =\text { the index } \lambda \text { of the FMRA. }
\end{aligned}
$$

Proof. Note that, by (5) and (10), $\lambda_{1}=p$ where $p$ is defined as in Definition 1.1(ii). Equations (2) and (15) imply that $\lambda_{1}=\max \left\{i:\left|\Delta_{i}\right|>0\right\}$, where $\left|\Delta_{i}\right|$ denotes the Lebesgue measure of $\Delta_{i}$. Recall that $\hat{V}_{1 \| x}=\hat{V}_{0 \| x} \oplus \hat{W}_{0 \| x}$ a.e. and that $\operatorname{dim} \hat{V}_{0|| x}=\chi_{\sigma\left(V_{0}\right)}(x)$. Hence $\operatorname{dim} \hat{W}_{0|| x}=\operatorname{dim} \hat{V}_{1|| x}-\operatorname{dim} \hat{V}_{0 \| x}=$ $\operatorname{dim} \hat{V}_{1 \| x}-\chi_{\sigma\left(V_{0}\right)}(x)$, which implies the first equality of (20). The second equality of (20) follows by the facts that

$$
2 \mathcal{Q} \cap \mathbb{T}^{d}=\sigma\left(V_{0}\right)^{c}, 2 B_{p} \cap \mathbb{T}^{d}=\Delta_{\lambda_{1}},
$$

where $\mathcal{Q}$ and $B_{p}$ are defined as in Definition 1.1 .

We now give a concrete construction of $\operatorname{such}\left\{\psi_{i}: 1 \leq i \leq \lambda\right\}$ based on Theorem 2.2, which recovers Theorem 1.2.

Theorem 2.5. Let $\left\{V_{j}\right\}_{j \in \mathbb{Z}}$ be an FMRA for $L^{2}\left(\mathbb{R}^{d}\right)$ and $\lambda:=\operatorname{len} W_{0}$. Then there exist $\left\{\psi_{l}\right\}_{l=1}^{n} \subset W_{0}$ such that $\left\{T_{k} \psi_{l}: 1 \leq l \leq n, k \in \mathbb{Z}^{d}\right\}$ is a tight frame for $W_{0}$ with the frame bound one if and only if $n \geq \lambda$.

Proof. If $n<$ len $W_{0}$, then the shifts of $n$ functions are not even complete in $W_{0}$ by the very definition of the length of the shift-invariant space. On the other hand, we now suppose that $n \geq \lambda$. Our goal is to construct a 1-periodic matrix-valued measurable mapping $M(x)$ of size $(\lambda+1) \times 2^{d}$ such that its first row of $M(x / 2)$ is $\left(m_{0}\left(\frac{x+q_{j}}{2}\right)\right)_{j=1}^{2^{d}}$ and it satisfies Conditions (1) and (2) 
of Theorem 2.2. The remaining entries of $M(x / 2)$ will be measurable simple functions in our constructions below. Recall

$$
\sigma\left(V_{1}\right)=\Delta_{2^{d}} \uplus \biguplus_{l=1}^{2^{d}-1} \biguplus_{1 \leq k_{1}<k_{2}<\cdots<k_{l} \leq 2^{d}} \Delta_{2^{d}-l}^{k_{1}, k_{2}, \ldots, k_{l}},
$$

where some of the sets may be null sets. Recall also that either $a_{x, q}=0$ or $\left\|a_{x, q}\right\|_{\ell^{2}\left(\mathbb{Z}^{d}\right)}=1$ for each $q \in Q$ by (5) since we assumed that the shifts of $\varphi$ form a tight frame with the frame bound one for $V_{0}$.

Now for any $x \in \mathbb{T}^{d} \backslash \sigma\left(V_{1}\right)$, let the remaining $\lambda$ rows be 0 . It suffices to define the remaining $\lambda$ rows of $M(x / 2)$ for a.e. $x \in \sigma\left(V_{1}\right)$. Now we divide into two cases.

Case I: $\lambda=\lambda_{1}$, i.e., $\left|\Delta_{\lambda_{1}} \backslash \sigma\left(V_{0}\right)\right|>0$. First, suppose that $\lambda=\lambda_{1}=2^{d}$. Then, $\left\|a_{x, q}\right\|_{\ell^{2}\left(\mathbb{Z}^{d}\right)}=1$ for each $q \in Q$ and for a.e. $x \in \Delta_{2^{d}}$ by (9). If $x \in \Delta_{2^{d}} \backslash \sigma\left(V_{0}\right)$, then $m\left(\frac{x+q}{2}\right)=0$ a.e. for each $q \in Q$ by (7). Hence the first row of $M(x / 2)$ is 0 . We define the remaining $\lambda=2^{d}$ rows to be those of the $2^{d} \times 2^{d}$ identity matrix. Note that for a.e. $x$ in such set, whose Lebesgue measure is positive, the last $\lambda$ rows of $M(x / 2)$ are linearly independent. Hence, $M(x / 2)$ with the first row deleted times $\left(a_{x, q}\right)_{q \in Q}^{t}$ gives $\lambda$ linearly independent vectors of $\hat{V}_{1 \| x}$. On the other hand, if $x \in \Delta_{2^{d}} \cap \sigma\left(V_{0}\right)$, then the norm of the first row is 1 by (7) since $\left\|a_{x, q}\right\|_{\ell^{2}\left(\mathbb{Z}^{d}\right)}^{2}=1$ for each $q \in Q$. We define the last row of $M(x / 2)$ to be 0 and define the in-between $\lambda-1$ rows so that the first $\lambda$ rows of $M(x / 2)$ is a unitary matrix. This definition satisfies Condition (1) of Theorem 2.2.

Next, suppose that $\lambda=\lambda_{1}=2^{d}-l<2^{d}$ for some $l \in\left\{1,2, \ldots, 2^{d}-1\right\}$. Then

$$
\Delta_{\lambda_{1}}=\underset{1 \leq k_{1}<k_{2}<\cdots<k_{l} \leq 2^{d}}{\biguplus} \Delta_{2^{d}-l}^{k_{1}, k_{2}, \ldots, k_{l}} .
$$

If $x \in \Delta_{2^{d}-l}^{k_{1}, k_{2}, \ldots, k_{l}}$, then $a_{x, q}=0$ if $q \in\left\{q_{k_{1}}, \ldots, q_{k_{l}}\right\}$ and $\left\|a_{x, q}\right\|_{\ell^{2}\left(\mathbb{Z}^{d}\right)}=1$ if $q \in$ $Q \backslash\left\{q_{k_{1}}, \ldots, q_{k_{l}}\right\}$. For a.e. $x \in \Delta_{2^{d}-l}^{k_{1}, k_{2}, \ldots, k_{l}} \backslash \sigma\left(V_{0}\right)$, the first row of $M(x / 2)$ is 0 . We define the remaining $\lambda$ rows of $M(x / 2)$ so that the $k_{i}$-th column is 0 for each $i=1,2, \ldots, l$, and the deletion of the first row of $M(x / 2)^{k_{1}, \ldots, k_{l}}$ is the $\lambda_{1} \times \lambda_{1}$ identity matrix. Since $\left|\Delta_{\lambda_{1}} \backslash \sigma\left(V_{0}\right)\right|>0$, at least one of $\Delta_{2^{d}-l}^{k_{1}, k_{2}, \ldots, k_{l}} \backslash \sigma\left(V_{0}\right)$ is of positive Lebesgue measure. For this set the last $\lambda$ rows of $M(x / 2)$ are linearly independent. Moreover, $M(x / 2)$ with the first row deleted times $\left(a_{x, q}\right)_{q \in Q}^{t}$ gives $\lambda$ linearly independent vectors of $\hat{V}_{1|| x}$. Now if $x \in \Delta_{2^{d}-l}^{k_{1}, k_{2}, \ldots, k_{l}} \cap \sigma\left(V_{0}\right)$, the norm of the first row of $M(x / 2)$ with each the $k_{i}$-th element deleted is 1 by (7). We define the last row of $M(x / 2)$ to be 0 and the $\lambda-1$ number of in-between rows so that all the $k_{i}$-th columns of $M(x / 2)$ are 0 and the first $\lambda$ rows of $M(x / 2)^{k_{1}, \ldots, k_{l}}$ is a unitary matrix. This definition satisfies Condition (2) of Theorem 2.2.

We have defined $M(x / 2)$ for $x \in \Delta_{\lambda_{1}}$. In particular, for at least one choice of $1 \leq k_{1}<k_{2}<\cdots<k_{l} \leq 2^{d}, \Delta_{2^{d}-l}^{k_{1}, k_{2}, \ldots, k_{l}} \backslash \sigma\left(V_{0}\right)$ has positive Lebesgue 
measure, and for a.e. $x$ in such set $M(x / 2)$ with the first row deleted times $\left(a_{x, q}\right)_{q \in Q}^{t}$ gives $\lambda$ linearly independent vectors of $\hat{V}_{1|| x}$.

Now our job is to define $M(x / 2)$ on

$$
\Delta_{\lambda_{1}-l}=\biguplus_{1 \leq k_{1}<k_{2}<\cdots<k_{2^{d}-\left(\lambda_{1}-l\right)}} \Delta_{\lambda_{1}-l}^{k_{1}, \ldots, k_{2^{d}-\left(\lambda_{1}-l\right)}}
$$

for $l=1,2, \ldots, \lambda_{1}-1$ so that it satisfies Condition (2) of Theorem 2.2. This can be done by the above idea in the following way. First, define the last $l$ rows of $M(x / 2)$ to be 0 . We only need to define the second to the $\left(\lambda_{1}-l+1=\lambda-l+1\right)$-st rows of $M(x / 2)$. If $x \in \Delta_{\lambda_{1}-l}^{k_{1}, \ldots, k_{2^{d}-\left(\lambda_{1}-l\right)}} \backslash \sigma\left(V_{0}\right)$, then the first row is 0 . Define the $k_{1}, \ldots, k_{2^{d}-\left(\lambda_{1}-l\right)}$-th columns of $M(x / 2)$ to be 0 and define the remaining rows so that $\lambda-l$ in-between rows with

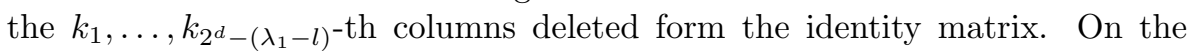
other hand, if $x \in \Delta_{\lambda_{1}-l}^{k_{1}, \ldots, k_{2^{d}-\left(\lambda_{1}-l\right)}} \cap \sigma\left(V_{0}\right)$, then the norm of the first row

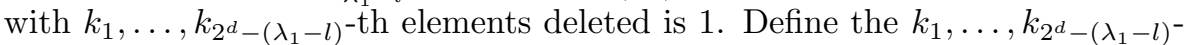
th columns of $M(x / 2)$ to be 0 and define $\lambda-l+1$-st row to be also 0 . Then we define the remaining $\lambda-l-1$ in-between rows so that the first $\lambda-l$ rows

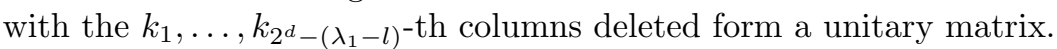

Case II: $\lambda=\lambda_{1}-1$, i.e., $\left|\Delta_{\lambda_{1}}\right| \sigma\left(V_{0}\right) \mid=0$. The proof is identical to Case I except for the fact that when we deal with $\Delta_{\lambda_{1}}$, the first row with suitable elements deleted is almost surely of norm 1 . Hence the construction yields a subset of $\Delta_{\lambda_{1}}$ of positive measure such that for a.e. elements of the set the first $\lambda_{1}=\lambda+1$ rows of $M(x / 2)$ with suitable columns deleted form a unitary matrix. The construction is identical for $\Delta_{\lambda_{1}-l}, 1 \leq l \leq \lambda_{1}-1$.

It is routine to see that $M(x / 2)$ satisfies Conditions (1) and (2) of Theorem 2.2 in either cases for a.e. $x \in \mathbb{T}^{d}$. Hence we have defined $M(x / 2)$ for a.e. $x \in \mathbb{T}^{d}$. If we scrutinize (13), then we see that we have defined the filters $m_{0}, m_{1}, \ldots, m_{\lambda}$ on $\mathbb{T}^{d}$. If we extend the filters 1-periodically, then we define a 1-periodic $M$. Now, finally, define $\psi_{1}, \psi_{2}, \ldots, \psi_{\lambda}$ so that (14) is satisfied. It is easy to see that $\psi_{1}, \psi_{2}, \ldots, \psi_{\lambda} \in L^{2}\left(\mathbb{R}^{d}\right)$. Then, Theorem 2.2 yields that $V_{1}=V_{0} \oplus W_{0}$, and $W_{0}=\oplus_{i=1}^{\lambda} \mathcal{S}\left(\left\{\psi_{i}\right\}\right)$. This completes the proof in the case that $n=\lambda$.

We now consider the case $n>\lambda$. Note that $\left|\Delta_{\lambda}\right|>0$. Then there exist positive measurable subsets $\left\{I_{i}\right\}_{i=0}^{n-\lambda}$ such that

$$
\Delta_{\lambda}=\uplus_{i=0}^{n-\lambda} I_{i}
$$

Define $M(x)$ as in Case I. Let $\tilde{M}(x)$ be the $n \times 2^{d}$ matrix-valued function defined by

$$
(\tilde{M}(x))_{i, j}= \begin{cases}(M(x))_{i, j}, & \text { if } 1 \leq i \leq \lambda-1 \\ (M(x))_{\lambda, j} \chi_{I_{i-\lambda}}(x), & \text { if } \lambda \leq i \leq n\end{cases}
$$


Define $\psi_{1}, \psi_{2}, \ldots, \psi_{n}$ so that (14) is satisfied by replacing $M(x)$ by $\tilde{M}(x)$. Using the argument similar to Case I, we can show that $\psi_{1}, \psi_{2}, \ldots, \psi_{n}$ satisfy that $V_{1}=V_{0} \oplus W_{0}$, and $W_{0}=\oplus_{i=1}^{n} \mathcal{S}\left(\left\{\psi_{i}\right\}\right)$.

We now give another interesting corollary to Theorem 2.2, which is related to the concept of the unitary extension principle (UEP) of Ron and Shen [15].

Corollary 2.6. Let $\psi_{0}:=\varphi \in L^{2}\left(\mathbb{R}^{d}\right)$ be refinable and its shifts form a tight frame with the frame bound one for $V_{0}:=W_{0}^{(0)}:=\mathcal{S}(\{\varphi\})$. Suppose there exist $n\left(\geq 2^{d}-1\right)$ functions $\psi_{1}, \psi_{2}, \ldots, \psi_{n} \in V_{1}:=D\left(V_{0}\right)$ such that the shifts of each $\psi_{l}$ form a tight frame with the frame bound one for $W_{0}^{(l)}:=\mathcal{S}\left(\left\{\psi_{l}\right\}\right)$ and (16) is satisfied. Then there exists a 1-periodic $(n+1) \times 2^{d}$ matrix-valued mapping $M$ satisfying (14) such that $M(x)^{*} M(x)=I_{2^{d}}$ for a.e. $x \in \sigma\left(V_{0}\right)$. In particular, if $\lim _{x \rightarrow 0} \hat{\varphi}(x)=1$, then $\left\{D^{j} T_{k} \psi_{l}: j \in \mathbb{Z}, k \in \mathbb{Z}^{d}, 1 \leq l \leq n\right\}$ is a tight frame associated with an FMRA defined by the UEP.

Proof. The hypotheses guarantee a 1-periodic $(n+1) \times 2^{d}$ matrix-valued mapping $M(x)$ satisfying (14). We change the entries of $M(x)$ so that (14) still holds and $M(x)^{*} M(x)=I_{2^{d}}$ for a.e. $x \in \sigma\left(V_{0}\right)$. First we need an obvious observation: If $N$ is an $(n+1) \times 2^{d}$ matrix such that whose $2^{d}$ rows are orthonormal and the remaining rows are 0 , then $N^{*} N=I_{2^{d}}$. This holds since the matrix obtained by deleting all the 0 rows are $2^{d} \times 2^{d}$ unitary matrix and since $N^{*} N$ is simply the product of the adjoint of the deleted matrix and the deleted matrix.

Suppose $x \in \sigma\left(V_{0}\right)$. Then [7, Lemma 8] shows that $\sigma\left(V_{1}\right)=2 \sigma\left(V_{0}\right)(\bmod 1)$. Hence there exists $k \in \mathbb{Z}^{d}$ such that $y:=2 x+k \in \sigma\left(V_{1}\right)$. Note that if we permute the columns of $M(y / 2)$ suitably, then $M(y / 2)$ becomes $M(x)$ since $y / 2=x+k / 2$. If $y \in \Delta_{2^{d}}$, then $M(y / 2)^{*} M(y / 2)=I_{2^{d}}$ by Condition (1) of Theorem 2.2 and by the above observation. Hence $M(x)^{*} M(x)=I_{2^{d}}$ by the nature of the column permutation. Now suppose that $y \in \Delta_{2^{d}-l}^{k_{1}, \ldots, k_{l}}$. Then $0=a_{y, q_{k_{1}}}=\cdots=a_{y, q_{k_{l}}}$. Hence $k_{1}, \ldots, k_{l}$-th columns of $M(y / 2)$ have no contribution in (14). Moreover, $M(y / 2)^{k_{1}, \ldots, k_{l}}$ satisfies Condition (2) of Theorem 2.2. It is easy to see that we can change $k_{1}, \ldots, k_{l}$-th columns of $M(y / 2)$ so that exactly $2^{d}$ rows are orthonormal and the remaining rows are 0 . Hence $M(y / 2)^{*} M(y / 2)=I_{2^{d}}$ also by the above observation. Therefore, $M(x)^{*} M(x)=I_{2^{d}}$ by the nature of the column permutation. In particular, if $\lim _{x \rightarrow 0} \hat{\varphi}(x)=1$, then $\left\{D^{j} T_{k} \psi_{l}: j \in \mathbb{Z}, k \in \mathbb{Z}^{d}, 1 \leq l \leq n\right\}$ is a tight frame by $[15]$.

\section{References}

[1] J. J. Benedetto and S. Li, The theory of multiresolution analysis frames and applications to filter banks, Appl. Comput. Harmon. Anal. 5 (1998), no. 4, 389-427.

[2] J. J. Benedetto and O. M. Treiber, Wavelet frames: multiresolution analysis and extension principles, Wavelet transforms and time-frequency signal analysis, 3-36, Appl. Numer. Harmon. Anal., Birkhauser Boston, Boston, MA, 2001. 
[3] C. de Boor, R. DeVore, and A. Ron, The structure of finitely generated shift-invariant spaces in $L_{2}\left(R^{d}\right)$, J. Funct. Anal. 119 (1994), no. 1, 37-78.

[4] M. Bownik, The structure of shift-invariant subspaces of $L^{2}\left(R^{n}\right)$, J. Funct. Anal. 177 (2000), no. 2, 282-309.

[5] H. Helson, Lectures on Invariant Subspaces, Academic Press, New York, 1964.

[6] R.-Q. Jia, Shift-invariant spaces and linear operator equations, Israel J. Math. 103 (1998), 259-288.

[7] H. O. Kim, R. Y. Kim, and J. K. Lim, Local analysis of frame multiresolution analysis with a general dilation matrix, Bull. Austral. Math. Soc. 67 (2003), no. 2, 285-295.

[8] _ Internal structure of the multiresolution analyses defined by the unitary extension principle, J. Approx. Theory 154 (2008), no. 2, 140-160.

[9] H. O. Kim and J. K. Lim, On frame wavelets associated with frame multiresolution analysis, Appl. Comput. Harmon. Anal. 10 (2001), no. 1, 61-70.

[10] _ Applications of shiff-invariant space theory to some problems of multiresolution analysis of $L^{2}\left(\mathbb{R}^{d}\right)$, in: D. Deng, D. Huang, R.-Q. Jia, W. Lin and J. Wang (Eds.), Studies in Advanced Mathematics 25: Wavelet Analysis and Applications, American Mathematical Society/International Press, Boston 2001, 183-191.

[11] J. K. Lim, Gramian analysis of multivariate frame multiresolution analyses, Bull. Austral. Math. Soc. 66 (2002), no. 2, 291-300.

[12] S. Mallat, Multiresolution approximations and wavelet orthonormal bases of $L^{2}(R)$, Trans. Amer. Math. Soc. 315 (1989), no. 1, 69-87.

[13] L. Mu, Z. Zhang, and P. Zhang, On the higher-dimensional wavelet frames, Appl. Comput. Harmon. Anal. 16 (2004), no. 1, 44-59.

[14] A. Ron and Z. Shen, Frames and stable bases for shift-invariant subspaces of $L_{2}\left(\mathbf{R}^{d}\right)$, Canad. J. Math. 47 (1995), no. 5, 1051-1094.

[15] _ Affine systems in $L_{2}\left(\mathbf{R}^{d}\right)$ : the analysis of the analysis operator, J. Funct. Anal. 148 (1997), no. 2, 408-447.

HONG OH KIM

Department of Mathematical Sciences

Korea Advanced Institute of Science and Technology

DAEJEON 305-701, KorEA

E-mail address: kimhong@kaist.edu

Rae Young Kim

Department of Mathematics

YEUNGNAM UNIVERSITY

GyeOngsan 712-749, KoreA

E-mail address: rykim@ynu.ac.kr

JAE Kun Lim

Department of Applied Mathematics

HANKYONG NATIONAL UNIVERSITY

AnseOng 456-749, KoreA

E-mail address: jaekun@hknu.ac.kr 